\title{
EL EMPLEO CONCEPTO GENTRIFICACIÓN EN EL CENTRO HISTÓRICO DE LA CIUDAD DE MÉXICO. \\ Un análisis comparativo de estudios recientes para discutir su pertinencia 2008- 2018.
}

\author{
Ivonne Guadalupe Garza Rodríguez \\ Universidad Nacional Autónoma de México UNAM \\ Director de tesis: Dr. Raúl Salas Espíndola \\ ivonnegpe.garza@gmail.com
}

\section{RESUMEN}

Desde la aplicación de políticas de corte neoliberal que procuran el mejoramiento de la Imagen Urbana del Centro Histórico de la Ciudad de México, en el 2000, se ha incrementado el número de publicaciones locales que afirman la presencia de gentrificación en la zona, a pesar de que el empleo del concepto ha sido debatido globalmente por su ambigüedad y falta de rigor académico.

Al encontrar falencias en estas publicaciones y contradicciones con la realidad observada se analiza el origen de las inconsistencias de las publicaciones locales, se cuestiona la pertinencia de su empleo en este contexto. Así mismo se explican las probables causas de su adopción y difusión.

Palabras clave: gentrificación, + pertinencia, + Centro Histórico de Ciudad de México

\begin{abstract}
Since the application of neoliberal policies that seek to improve the Urban Image of the Historic Center of Mexico City, in 2000, the number of local publications that affirm the presence of gentrification in the area has increased, despite that the use of the concept has been debated globally due to its ambiguity and lack of academic rigor.
\end{abstract}

When finding shortcomings in these publications and contradictions with the observed reality, the origin of the inconsistencies of the local publications is analyzed, the applicability of their use in this context is questioned. Likewise, the probable causes of their adoption and diffusion are explained.

Keywords: gentrification, + applicability, + Historical Center of Mexico City 


\section{INTRODUCCIÓN}

En las últimas décadas los centros históricos latinoamericanos han sido objeto de intervenciones que buscan mejorar la imagen urbana para la protección del patrimonio histórico. Estas acciones han tenido consecuencias como: el incremento del costo del suelo, el cambio de giro en establecimientos comerciales, la segregación social, la exclusión, expulsión y desalojo de los sectores sociales desfavorecidos. Estos fenómenos han sido interpretados por la academia y una parte de la sociedad como gentrificación: un concepto polisémico e indeterminado que ha sido utilizado para describir mejoras en el entorno urbano en áreas con potencial de lucro, y que van acompañadas de la sustitución de la población desfavorecida por una de mayor poder adquisitivo, el incremento del precio del suelo o el cambio de uso para obtener mayores beneficios económicos.

En Ciudad de México a partir del 2000 se registra un incremento en las investigaciones que determinan la existencia de gentrificación en ciertas zonas -entre ellas el Centro Histórico-; sin embargo, existen contradicciones entre las publicaciones que afirman la presencia de gentrificación y la realidad observada, ya que esta última no presenta las características de este proceso según la literatura que aborda la temática. EI objetivo de este artículo es discutir la pertinencia del empleo del concepto gentrificación en el contexto del Centro Histórico de Ciudad de México, ya que este concepto polisémico e indeterminado, además de generar confusión, puede ser utilizado para soslayar o malinterpretar otras problemáticas sociales que están latentes y requieren atención. Para ello, en primer lugar, se expone un resumen del análisis de aproximadamente 25 publicaciones locales elaboradas entre los años 2008 y 2017, la caracterización de las falencias detectadas y una explicación de las causas que pudieron originarlas; en segundo lugar, se exponen dos casos de estudio en los que se evidencian las contradicciones entre las publicaciones y la realidad concreta; finalmente se exponen los argumentos de teóricos como Gramsci y Bourdieu para explicar por qué se emplea el concepto, a pesar de que no se observan sus características en la realidad concreta.

\section{LAS PUBLICACIONES LOCALES: LAS FALENCIAS Y EL ANÁLISIS DE SUS PROBABLES CAUSAS}

Las publicaciones sobre gentrificación en Ciudad de México se han incrementado notoriamente en los últimos años; una muestra de ello es que en la base de datos de la Universidad Nacional Autónoma de México las tesis que contienen en sus títulos los términos: gentrificación y Ciudad de México tienden al alza: la primera tesis publicada data del año 2008, en 2013 aparece la segunda, para el 2014 se publicaron tres, en 2015 cuatro, en 2016 cinco y finalmente, hasta marzo de 2017 existían tres, lo que suma un total de 17 tesis publicadas en 8 años sobre la temática (UNAM, 2017).

Además, se encontró una serie de artículos científicos y publicaciones sobre gentrificación en las zonas centrales de la ciudad, escrita a partir de la aplicación de las políticas públicas de revitalización del Centro Histórico realizadas entre los años 2000 y 2010, acerca de las mejoras urbanas y arquitectónicas promovidas para proteger el Patrimonio Cultural y fomentar la industria turística.

Al revisar aproximadamente 25 publicaciones locales -incluidos algunos trabajos de titulación de jóvenes investigadores-, elaboradas entre 2014 y 2017 se encontró dos tipos de irregularidades generales: en primer lugar, que hacían alusión a fenómenos de carácter social y urbano con características diferentes para referirse a la gentrificación; en segundo lugar se detectaron contradicciones internas, conclusiones con evidencias insuficientes o sin presentar datos contundentes, y otras con limitantes metodológicas para comprobar la presencia de algunas variables relacionadas con la gentrificación.

Para analizar las publicaciones en busca de los diversos fenómenos sociales y urbanos a los que se alude cuando se habla de gentrificación, se procedió a distinguirlos y clasificarlos según las características mencionadas en cada texto. Cabe mencionar que se presentaron dificultades al momento de distinguirlos ya que estaban interconectados entre sí, yuxtapuestos o algunos de ellos implícitos en las descripciones a pesar de no estar señalados en los trabajos; por ello se optó por considerar únicamente los fenómenos que los autores mencionan en las publicaciones y definen los resultados obtenidos.

En las publicaciones se hace alusión a los siguientes fenómenos sociales y urbanos, así como algunas de sus características, para indicar la presencia del proceso;

- La intervención del Gobierno como detonador del proceso: mediante políticas públicas de corte neoliberal que promueven la conservación del patrimonio histórico para revitalizar las zonas, fomentar 
su renovación y redesarrollo, y beneficiar al sector turístico (Martínez, 2014; Olivera \& Delgadillo, 2014; Zamora, 2014).

- El cambio en el uso de suelo que favorece la gentrificación: en estas investigaciones se exponen algunas discordancias e irregularidades entre los Planes de Desarrollo Urbano y los usos del suelo en la actualidad (Salinas, 2013; López, 2016).

- Cambios en la imagen urbana: construcción de edificios habitacionales en zonas céntricas, peatonización de calles, conservación y mejoramiento de áreas verdes, limpieza, vigilancia, etc. (Salinas, 2013; Martínez, 2014; Pérez, 2016; López, 2017).

- Incremento en el precio del alquiler y del suelo: Díaz (2014), Cordero (2015), López (2016) y Romero (2017) señalan que, después de las mejoras urbanas y arquitectónicas ejecutadas en el Centro Histórico, se ha incrementado el costo del suelo. Algunos de ellos mencionan que primero hubo una etapa de desvalorización -por el congelamiento de rentas y el sismo de 1985- que precedió a la revalorización de las zonas centrales por parte del Gobierno y agentes privados, por lo que existiría "Rent-Gap" o escalón diferencial de renta.

- La sustitución de una población desfavorecida por otra con mejor cualificación socioeconómica: Salinas (2013), Díaz (2014), Bournazou (2015), Mondragón (2015), Cordero (2015), López (2016), Rodríguez (2016), Gutiérrez (2016), entre otros indican en sus estudios la existencia de este cambio en el perfil socioeconómico de la población.

Zamora (2014), Martínez (2014), Tellez (2014), Pérez (2016) y Rodríguez (2016), describen un cambio en las relaciones sociales en los barrios y las calles causadas por el establecimiento de otros giros comerciales, el arribo de nueva población flotante, la creación de museos o recintos con fines culturales, o inclusive por la peatonización de calles. Algunos señalan que ahora son espacios "mercantilizados" es decir, que cualquier actividad que se lleve a cabo en el sitio implica la posibilidad del consumo de un bien o servicio.

Se mencionan tambien: la inserción de grupos de artistas para cambiar la configuración del barrio, la estigmatización de ciertas zonas para su posterior desvalorización y probable revalorización, el desplazamiento -en forma física o simbólica- de la población que habita (o habitaba) el sitio, las resistencias sociales ante este proceso, etc.

Después de analizar estas publicaciones se detectó que el concepto gentrificación no permite explicar ni describir los acontecimientos particulares que se presentan en las zonas de estudio y que existen imprecisiones en las conclusiones por la falta de rigor metodológico. También se evidenció que algunas publicaciones presentan contradicciones internas: básicamente, los autores definen las variables de la gentrificación en su marco teórico, realizan estudios de campo, no encuentran evidencia empírica para sostener la existencia del fenómeno, pero concluyen que sí existe gentrificación.

Otras investigaciones presentan evidencias insuficientes o no presentan datos contundentes: no se indica el número o porcentaje de población entrevistada ni el universo considerado; en otras se afirma la presencia de gentrificación sin que las conclusiones sean determinantes: "Por lo tanto hay gentrificación, aunque tenue, que implica cierto grado de sustitución" (Díaz, 2014:9).

Finalmente, que la recopilación de datos es un reto, muchas veces insalvable para los investigadores a pesar de que mantengan el rigor metodológico, porque las instituciones encargadas de levantar la información han modificado su nivel de agregación geográfica en los últimos años, o porque no permiten el acceso a cierta información -por la ley de protección de datos personales-. Se observó en estas publicaciones que, tanto los jóvenes investigadores como los expertos obtienen del marco teórico de la gentrificación las variables que consideran pertinentes para sostener su postura; que los jóvenes investigadores tienden a innovar metodologías mediante estrategias mixtas conduzcan a los resultados previstos, y que los autores con mayor experiencia emplean metodologías probadas previamente en otras regiones geográficas.

Después de analizar las publicaciones se observa que el proceso de investigación se hace a la inversa: primero se busca encontrar las variables y características ceñidas al marco teórico de la gentrificación para determinar su existencia, en lugar de describir y explicar libremente los fenómenos que acontecen y después cuestionar la pertinencia del empleo del concepto para describir el fenómeno observado, lo que podría contribuir al desarrollo de teorías sobre gentrificación. Las falencias encontradas denotan cierta confusión en 
los jóvenes autores y un notorio interés, por parte de los investigadores más experimentados, en determinar la presencia o ausencia del fenómeno en ciertas áreas.

Una posible explicación para la confusión y las contradicciones que se hacen latentes en las investigaciones, es el carácter polisémico del concepto. Desde que se acuñó había fuertes cuestionamientos debido a su imprecisión, sobre todo temporal, ya que procesos similares de lucha entre clases por el dominio del territorio han estado presentes a lo largo de la historia en diferentes regiones. Sin embargo, Neil Smith, uno de los autores legitimados que abordan la temática, considera que estos son movimientos predecesores de la gentrificación, por lo que una gran cantidad de investigadores lo avalan.

A continuación, se muestran los diversos significados que se han otorgado a la gentrificación a través del tiempo; se busca sostener que éste depende de la postura de cada autor y de las particularidades culturales y económicas de cada contexto geográfico, por lo que sería ilógico adoptarlo para describir la realidad en un contexto diferente al de su enunciación; también se enlistan las características más usuales que se otorgan a la gentrificación.

\subsection{Gentrificación: un concepto polisémico e indeterminado (Características comunes del proceso)}

El concepto fue acuñado por la socióloga Ruth Glass en 1964 para referirse al proceso de renovación de las viviendas de la zona central de Londres y la sustitución de la población obrera por una clase con mejores ingresos económicos que la anterior, y que se dedicaba a otras actividades -servicios- propias de la época postindustrial (Glass, 1964).

Posteriormente otros autores retomaron el concepto para describir fenómenos que tenían cierta semejanza a los procesos mencionados por Ruth Glass y lo aplicaron en sus países; en un inicio se hizo hincapié en las peculiaridades que el fenómeno presentaba en cada región, pero a pesar de ello, todos fueron considerados como gentrificación. Es así como el concepto se expandió rápidamente a Holanda, Alemania, Australia, Canadá y Estados Unidos.

En el caso de este último país, Downs (1981, citada en Beauregard 1990:885) señala que las investigaciones que ya existían sobre los cambios en los vecindarios durante el periodo de desindustrialización -como los de Hoover y Vernon (1959, citados en ibíd.) o Birch (1971, citado en ibíd.), que refieren a la declinación de algunos barrios y las posibilidades de revertir este proceso a través de la construcción de apartamentos- son descartadas para dar lugar a nuevas investigaciones basadas en la gentrificación, con lo cual pudo perderse la posibilidad de ensayar nuevas explicaciones basadas en el estudio de la realidad y no en un concepto importado de Europa.

Tanto en Estados Unidos como en Canadá abundaron los estudios sobre gentrificación entre 1970 y 1985; en la mayor parte de los casos el objetivo fue describir el proceso y encontrar las causas que lo originaban (Vázquez, 1996). A partir de ese momento el concepto adquirió diferentes definiciones: hubo quienes observaban en él cualidades positivas y otros que resaltaron sus consecuencias negativas. Por ejemplo, David Ley, en Canadá, determinó que la causa principal de la gentrificación -entendida como la sustitución de población de bajos ingresos por otra, nacida del "baby boom" de la Segunda Guerra Mundial, con un alto nivel educativo, mejor cualificación laboral y mayores ingresos que la población anterior- es la fuerte demanda de vivienda en las zonas centrales, que ocasiona el incremento del precio del suelo y las modificaciones en el entorno.

Al observar la gentrificación como consecuencia de la demanda de espacios centrales por personas con mayor poder adquisitivo y mejor cualificación laboral, surgen definiciones como las de Caulfield (1989) para quien la "gentrificación es un proceso que une a las personas en la ciudad central y crea oportunidades para la interacción social, la tolerancia y la diversidad cultural" y que "[...]la gentrificación es una experiencia liberadora para los gentrificadores y aquellos que entran en contacto con ellos" (Lees, Slater, y Wyly, 2008:209). Inclusive el proceso llega a interpretarse como una reacción a las instituciones represivas presentes en los suburbios: los gentrificadores abrirían las puertas a sectores anteriormente discriminados los homosexuales o las madres solteras-, quienes abrirían el camino para los desarrolladores y crearían las condiciones para la apertura de cafés, restaurantes, áreas verdes, etc. ${ }^{1}$

\footnotetext{
${ }^{1}$ Para Moore (1982) "la gentrificación representa un proceso mediante el cual un grupo importante de la nueva clase social emergente establece una identidad residencial concomitante con su identidad social, en el contexto de unos cascos urbanos paulatinamente convertidos en la residencia de los denominados trabajadores de cuello blanco" (Rodríguez y Puga, 2002: 280).
} 
En cambio, Neil Smith (1979) en Estados Unidos observó que, después de la devastación de la guerra, la élite inversionista aprovechó los precios bajos del suelo urbano ocupado por una clase desfavorecida para comprar inmuebles, hacer intervenciones urbano-arquitectónicas y venderlos después a un precio mucho más alto. Por ello, centró su atención en la relación entre el valor del suelo y el de los inmuebles, desarrollando la teoría del "rent gap".2

Considerar la gentrificación como consecuencia de la oferta generada por el Estado y agentes financieros implica que una élite gobernante y un sector social desfavorecido luchan por el dominio del territorio, lo que a su vez impacta en la capitalización del suelo. Esta línea teórica ha sido comprobada empíricamente por múltiples autores a través del tiempo en diferentes geografías; por ejemplo, Ludek Sykora (1993) sobre la transición del mercado y la aparición de un aumento de precios del suelo en Praga, o los estudios de Blair Badcock en Adelaida, Eric Clark en Malmö, Dan Hammel en Minneapolis y David O. Sullivan en el este de Londres. Cada uno proporciona pruebas de que la trayectoria de las rentas capitalizadas y potenciales del terreno sí sigue la tendencia general teorizada por Neil Smith (Lees, Slater, y Wyly, 2008:62-66).

Este diferencial de renta se expresaría de forma diferente en el paisaje urbano, dependiendo del tipo de reglas que gobiernan un mercado inmobiliario específico, los reglamentos de zonificación, las tasas impositivas para los diferentes usos de la tierra, los incentivos fiscales diseñados para fomentar el redesarrollo y otros factores que contribuyen a dar forma a la valorización de una ciudad, en un contexto regional y nacional particular, así como las costumbres o preferencias culturales de la población (Lees, Slater, y Wyly, 2008:70).

No solo en estas dos posturas se le otorga un significado al concepto de gentrificación; otras definiciones surgen al intentar conciliarlas. Por ejemplo, Hamnett (1984) define la gentrificación como: "Un fenómeno a la vez de tipo físico, económico, social y cultural. La gentrificación habitualmente implica la invasión, por parte de clases medias y grupos con elevados ingresos, de vecindarios o áreas en decadencia y la reubicación o desplazamiento de gran parte de sus ocupantes originarios. Ello implica la renovación física de lo que frecuentemente es un stock de vivienda en grave estado de abandono y su renovación o mejora a fin de satisfacer las demandas de sus nuevos ocupantes. En este proceso, el parque de viviendas afectado haya sido o no renovado, experimenta un significativo aumento de precios. Además, este tipo de procesos de cambio incluye habitualmente cierto grado de transformación del régimen de tenencia pasando del alquiler a la propiedad" (Vázquez,1996:10).

Desde 1990 la gentrificación se consideró una consecuencia del desarrollo urbano global: Lees, Slater y Wyly (2008) aseveran que, tanto los académicos como los analistas políticos, observan el proceso de gentrificación como una de las "consecuencias de la desregulación, la comercialización y privatización, del mercado de la vivienda" (Lees, Slater y Wyly, 2008:163-165). Loreta Lees en el 2000 observa que la gentrificación ahora está determinada por los procesos urbanos a gran escala -relacionados con la economía global- donde una clase con mucho mayor poder adquisitivo -los "ultra-gentrificadores"- reemplaza a los primeros gentrificadores, por lo que habla de una "súper- gentrificación" (Brown - Saracino, 2010:48). ${ }^{3}$ De la misma forma Atkinson y Bridge (2000, citados en Brown - Saracino, 2010:56-57) vinculan el proceso con el concepto de ciudad global de Sassen: los inversionistas de talla internacional compran tierras en centros importantes de diferentes países, tanto económica como políticamente; el encarecimiento consecuente de las viviendas y/o servicios desplazaría paulatinamente a los habitantes locales.

En las últimas décadas muchos de los autores dejaron de lado las explicaciones estructurales de las causas del proceso -asumiéndolas como posturas "clásicas" de la gentrificación-, para enfatizar otros ejes temáticos en la denominada "postgentrificación": la vivienda deja de ser uno de los elementos centrales y empiezan a surgir diferentes derivaciones del concepto. Lees, Slater, y Wyly (2008:129-136) sugieren que la primera derivación fue la gentrificación rural, descrita por Parsons en 1980: estudia el vínculo entre el asentamiento de las nuevas clases medias, las transformaciones socioeconómicas y culturales del paisaje rural y el consecuente desplazamiento y marginalización de los grupos de bajos ingresos. Es similar a la greentrification, propuesta por Smith y Phillips (2001) para describir la problemática existente en las áreas rurales consideradas como atractivos para el turismo.

\footnotetext{
${ }^{2}$ Si los inmuebles se deprecian al punto de que su valor de renta de capitalización es menor que el valor potencial del uso del suelo en sus "mejores posibilidades de uso", ello produce un "desequilibrio de renta". Cuando éste es lo bastante grande para asegurar el beneficio se genera la gentrificación: "proceso por el cual un vecindario ocupado por una clase trabajadora es rehabilitado por los desarrolladores, profesionistas y compradores -pertenecientes a la nueva clase media-" El autor aclara que es diferente el redesarrollo que la gentrificación puesto que [...] El redesarrollo no incluye la rehabilitación de las viejas estructuras, pero sí la construcción de nuevos edificios en una tierra previamente urbanizada" (Smith, 1982, citado en Davidson y Lees, 2005:1166).

${ }^{3}$ Este concepto es muy similar al "desplazamiento exclusionario", donde las nuevas viviendas no son asequibles para los ciudadanos de clases más bajas (Duque: 2010).
} 
Se encuentran derivados como: Studentification, acuñado por Darren Smith en 2002, para describir los procesos sociales, ambientales y económicos causados por el gran número de estudiantes que invaden ciertas áreas de las ciudades y pueblos donde se sitúan las universidades; la gentrificación comercial, boutiqueification, o retail gentrification, que se refiere al cambio de giro en los comercios -cafés, restaurantes, bares, galerías de arte y artesanías exclusivas, etc.- para responder a la demanda de una clase con mayor poder adquisitivo que ahora reside en estos lugares, abordada por Sharon Zukin en 1990, David Ley en 1996, Dowling en 2001, entre otros.

La gentrificación turística -derivación de la gentrificación usada por Gotham en 2005- se usa para referirse a la transformación de un vecindario en un enclave que incorpora el entretenimiento y el turismo con actividades orientadas al consumo. Aquí están involucradas tanto las industrias culturales como los capitalistas; en este sentido es relativa tanto a la gentrificación comercial como a la residencial; la gentrificación provincial (Lees, 2000; Smith, 2001) refiere a procesos concretos de ciudades pequeñas generados por los gobiernos locales, pues se busca atraer a las personas de clase media al interior de las provincias y mantenerlas ahí.

Existen otras derivaciones que van más allá del aspecto tangible; está la gentrificación simbólica en Latinoamérica, que se refiere a la implantación violenta de otros valores culturales y costumbres que excluyen a parte de la población local, debido a los cambios en las dinámicas sociales en sus vecindarios (Hiernaux y González, 2014).

Las derivaciones del concepto explicadas anteriormente -a excepción de la última- tienen algunas características en común: la sustitución de la población de una clase desfavorecida por otra de un mayor cualificación y poder adquisitivo; el incremento en los precios del suelo en las ciudades en las que se llevan a cabo operaciones financieras de primer nivel -Nueva York, Londres, Tokio, etc.-; la participación del Estado como un instrumento que promueve los procesos de gentrificación, mediante la implementación de políticas públicas para la generación de espacios verdes, peatonización de calles, mejoramiento en la infraestructura urbana, los cambios en los usos de suelo y su nivel de ocupación, el fomento a la industria turística, entre otros; la segregación socio-espacial, que va desde la contratación de vigilancia y seguridad privada hasta la construcción de barreras físicas, como muros, que limitan el acceso a cierto sector de la población. Finalmente, a pesar de las diferencias entre diversos autores, se ha encontrado que la gentrificación lleva implícito el mejoramiento físico de un inmueble o del entorno.

\section{EL EMPLEO DEL CONCEPTO GENTRIFICACIÓN EN EL CENTRO HISTÓRICO DE LA CIUDAD DE MÉXICO Y LAS DISCORDANCIAS CON LA REALIDAD OBSERVADA}

El empleo de este concepto se ha generalizado a tal grado que muchas de las acciones derivadas de las políticas públicas de rescate y revitalización del Centro Histórico de Ciudad de México implementadas a partir del 2000 -que han tenido como consecuencia la expulsión de vendedores ambulantes o de una clase desfavorecida, el incremento del precio del suelo, el cambio de uso de suelo para promover el crecimiento de la industria turística, ${ }^{4}$ son interpretadas como gentrificación, a pesar de que estas características podrían presentarse en cualquier proceso de renovación urbana de la época neoliberal en la que se busque capitalizar al máximo el uso del suelo.

Como ya se explicó antes, algunos académicos y jóvenes investigadores parecen esforzarse más en comprobar la existencia de gentrificación en estas zonas -mediante la presencia de las variables determinadas por autores legitimados internacionalmente por su trayectoria- que en explicar los fenómenos sociales y urbanos que se observan en la realidad concreta, que podrían contener particularidades aun no consideradas a través de las cuales contradecir la presencia del proceso en la zona.

Los siguientes casos de estudio son ejemplos de sitios donde los autores locales han establecido la existencia de gentrificación, aunque muestren particularidades que no se esperarían de un sitio gentrificado.

\subsection{El caso de la Zona Sur de la Alameda Central}

\footnotetext{
${ }^{4}$ Por ejemplo: la restauración de inmuebles patrimoniales, habilitación de edificios de vivienda, restauración de fachadas, ampliación de una oferta cultural y el fomento a la industria turística con la creación y habilitación de museos y espacios destinados a exhibiciones artísticas o musicales, el incremento de restaurantes, cafés, hoteles y bares, la creación y remodelación de plazas y jardines públicos, la peatonización de calles, la creación de corredores de consumo especializados -Madero y Regina-, así como la implementación de seguridad pública, mecanismos de vigilancia y la liberación de comercios en vía pública mediante la reubicación de vendedores ambulantes en plazas comerciales.
} 
Ibán Díaz Parra en su publicación: La transformación del Centro Histórico de la Ciudad de México. ¿Gentrificación o repoblación?, señala que existen registros de que este sector, desde la segunda mitad del siglo XX, empezó a decaer debido a los procesos de despoblamiento, de desinversión gubernamental y los sismos del 1957 y 1985 que afectaron muchos inmuebles, además de que era un sitio estigmatizado por la presencia de vendedores ambulantes y la inseguridad. Esto cambió radicalmente para el año 2000, cuando fueron notorias las transformaciones físicas derivadas de la aplicación de políticas de rescate del Centro Histórico -implementadas desde mediados de la década de 1990-, que tenían como objetivo conservar el patrimonio y dinamizar la economía mediante el turismo a través de esquemas de inversión público-privadas.

El objetivo de este autor es determinar la promoción social de la población desfavorecida y su sustitución por una clase social de mayores ingresos en el Centro Histórico de Ciudad de México. Para ello emplea una versión clásica y restrictiva del concepto gentrificación: "aburguesamiento residencial de los sectores urbanos humildes o en declive, implicando tanto la sustitución de residentes y usuarios como la reinversión del capital fijo en diferentes formas. En términos ecológicos se trataría de una sustitución de un hábitat social por otro de carácter más pudiente" (Díaz, 2014:2).

Para cumplir el objetivo emplea algunos datos estadísticos de otras investigaciones locales y los censos 2000, 2005 y 2010; utiliza las variables del número de población y el nivel de estudios, y las correlaciona con la influencia de las políticas públicas para la repoblación de esta zona para indicar que: "En el centro histórico, fuera del perímetro A, aunque limítrofe al mismo, es notorio el cambio de los polígonos al sur de la Alameda, de encontrarse por debajo de la media a estar por encima en 2010. Dentro del perímetro A, esto también ocurre con algunos sectores en el extremo suroeste del mismo." (ibid: 7-8).

Con base en lo anterior concluye que: [...] "En el suroeste del centro histórico ha habido una promoción social relativa entre 2000 y 2010, pero entre esos dos stocks la población no solo no había crecido, sino que se había reducido ligeramente. Por lo tanto, parece lógico pensar que durante el periodo estudiado ha salido población tanto como ha entrado otra nueva y de perfil radicalmente diferente. Tampoco es sostenible afirmar que la promoción social relativa de estos espacios sea producto de procesos de promoción social internos, como si estos pudieran ser independientes del resto de población de la urbe. Por lo tanto, hay gentrificación, aunque tenue, que implica cierto grado de sustitución. Comprobar los efectos negativos sobre las clases populares de este proceso, en términos de desplazamiento y desarticulación de su hábitat, implicaría un trabajo de campo en profundidad y el uso de técnicas cualitativas" (ibid.:9).

Estas conclusiones presentan imprecisiones: ¿cuánto es un tenue grado de sustitución de población?, ¿en qué medida esas únicas variables alcanzan para establecer con certidumbre las conclusiones? y finalmente, que los cambios en el nivel de estudios durante 10 años no necesariamente implican que exista nueva población con otro perfil socioeconómico ya que es probable que en ese tiempo la misma población alcanzara un mayor grado o nivel académico. Según lo concluido en este artículo se esperaría observar sustitución de población en la zona y cierta mejora en la imagen urbana, pero en la calle Artículo 123 , ubicada dos cuadras al sur de la Alameda Central es notoria la presencia de indigentes y falta de higiene en las calles.

Por otra parte, también existen trabajos de carácter cuantitativo realizados con mayor rigor metodológico; Eftychia Bournazou presenta en su publicación: "Cambios socioterritoriales e indicios de gentrificación. Un método para su medición" una serie de variables para determinar la presencia de gentrificación a nivel de la Ciudad de México. Esta autora también muestra la zona sur de la Alameda Central como un área gentrificada (ver gráfico 1); la metodología que emplea presenta algunas limitantes, por ejemplo: se señala la promoción social de la clase con menores ingresos en una escala geográfica de estudio muy grande, que abarca toda una AGEB, por lo que es imposible señalar a nivel de calle en donde está ubicada la promoción social; la temporalidad del estudio es solo de 10 años por lo que la autora no puede comprobar el destino de los desplazados (si es que los hubiera) ni los motivos del desplazamiento; las variables que emplea son insuficientes para indicar el perfil socioeconómico de la población; finalmente, no considera las particularidades de la población que vive y trabaja en el sitio, los intersticios legales que sirven a los habitantes para permanecer en el lugar, o las dinámicas sociales existentes. 


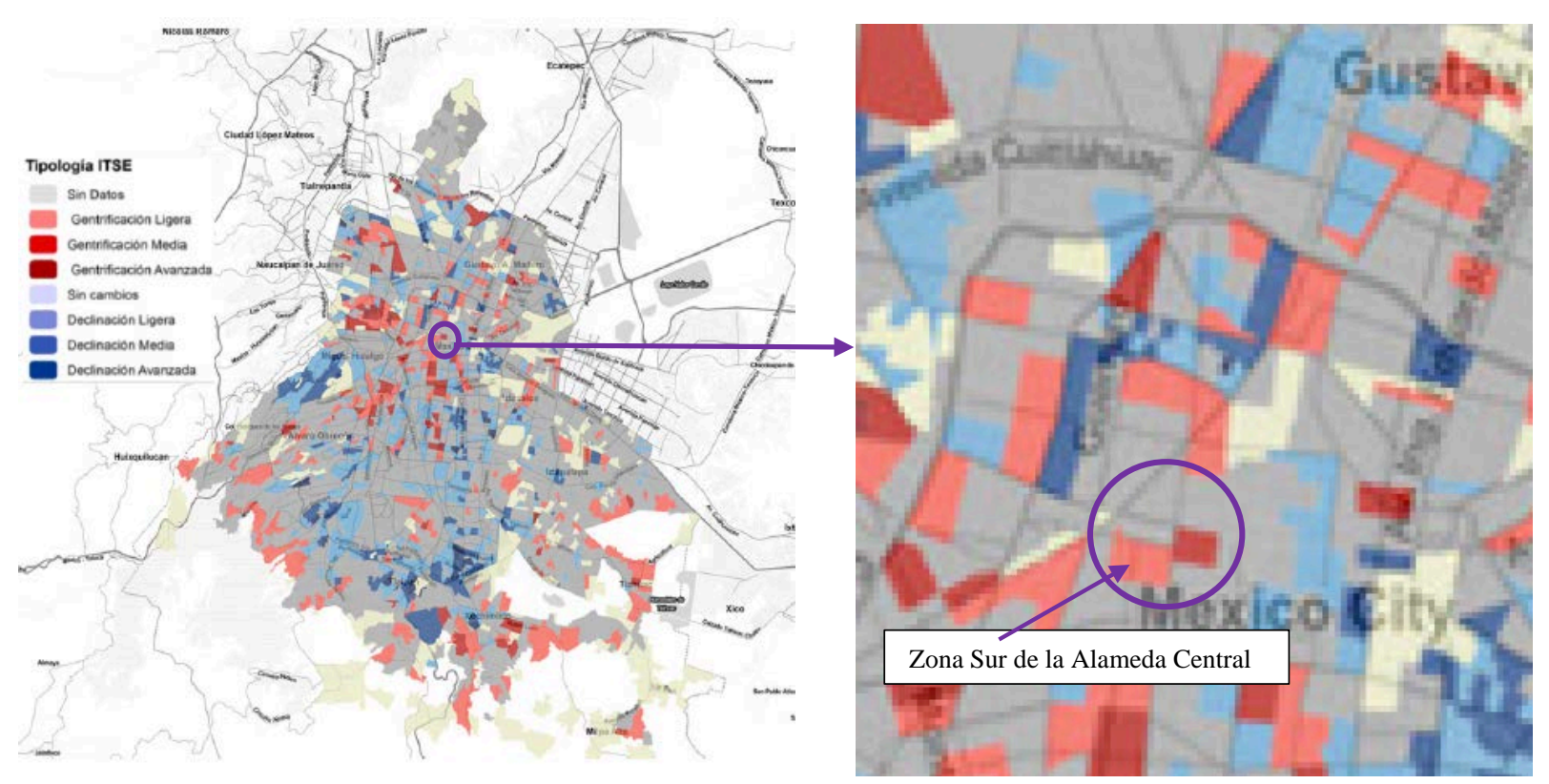

Gráfico 1. Mapa de Gentrificación en la Ciudad de México, periodo 2000-2010.

Fuente: Bournazou, E. (2015), "Cambios socio territoriales e indicios de gentrificación. Un método para su medición" Academia XXII 4759: UNAM.

La realidad concreta en la zona sur de la Alameda Central es más compleja de lo que se presenta en los estudios sobre gentrificación. Por ejemplo, en 2004 en la calle Artículo 123 esquina con Humboldt se construyó el desarrollo residencial "Living Centro Histórico", sobre un predio que anteriormente funcionaba como estacionamiento; en esa época pocos indigentes habitaban cerca del lugar. Siguiendo la lógica del mercado, los inversionistas se vieron motivados a construir el edificio por la demanda que prometía la zona una vez que se culminaran las mejoras, que inició el gobierno local en la Alameda y sus alrededores: la repavimentación de los andadores, la transformación y arreglo de los jardines, la expulsión de vendedores ambulantes y otra población no deseada de los perímetros, la creación de museos, el incremento en la oferta de servicios y, la implementación de vigilancia constante-. Esta inversión tal vez podría interpretarse como un inicio de gentrificación, a partir de la teoría del rent-gap desarrollada por Smith en 1979.

Sin embargo al mismo tiempo sucedían procesos alternos que modificaron estas condiciones: se ha documentado -en publicaciones hemerográficas y una entrevista realizada a uno de los inversionistas (Ing. Miguel Olvera, 2016) del nuevo desarrollo- que en 2010, una vez terminado el edificio y al tiempo que se ejecutaban las mejoras urbano-arquitectónicas en la Alameda y sus alrededores, se incrementó el número de indigentes alrededor del edificio porque esta población, expulsada de la Alameda Central, se instaló cerca del desarrollo habitacional (ver gráfico 2). Esto generó que la población de mayores ingresos no estuviera interesada en adquirir los inmuebles o vivir en el sitio, debido a la percepción de inseguridad y falta de higiene en la zona. Los inversionistas, con el apoyo de las autoridades locales, buscaron reubicar a los indigentes en un albergue construido especialmente para ellos, pero volvían al poco tiempo de ser reubicados; por lo tanto, se vieron forzados a bajar los precios de venta de los departamentos; esta otra parte de la realidad podría ser interpretada como degentrificación: ello contradice los estudios de Díaz Parra y Bournazou, reseñados antes. 


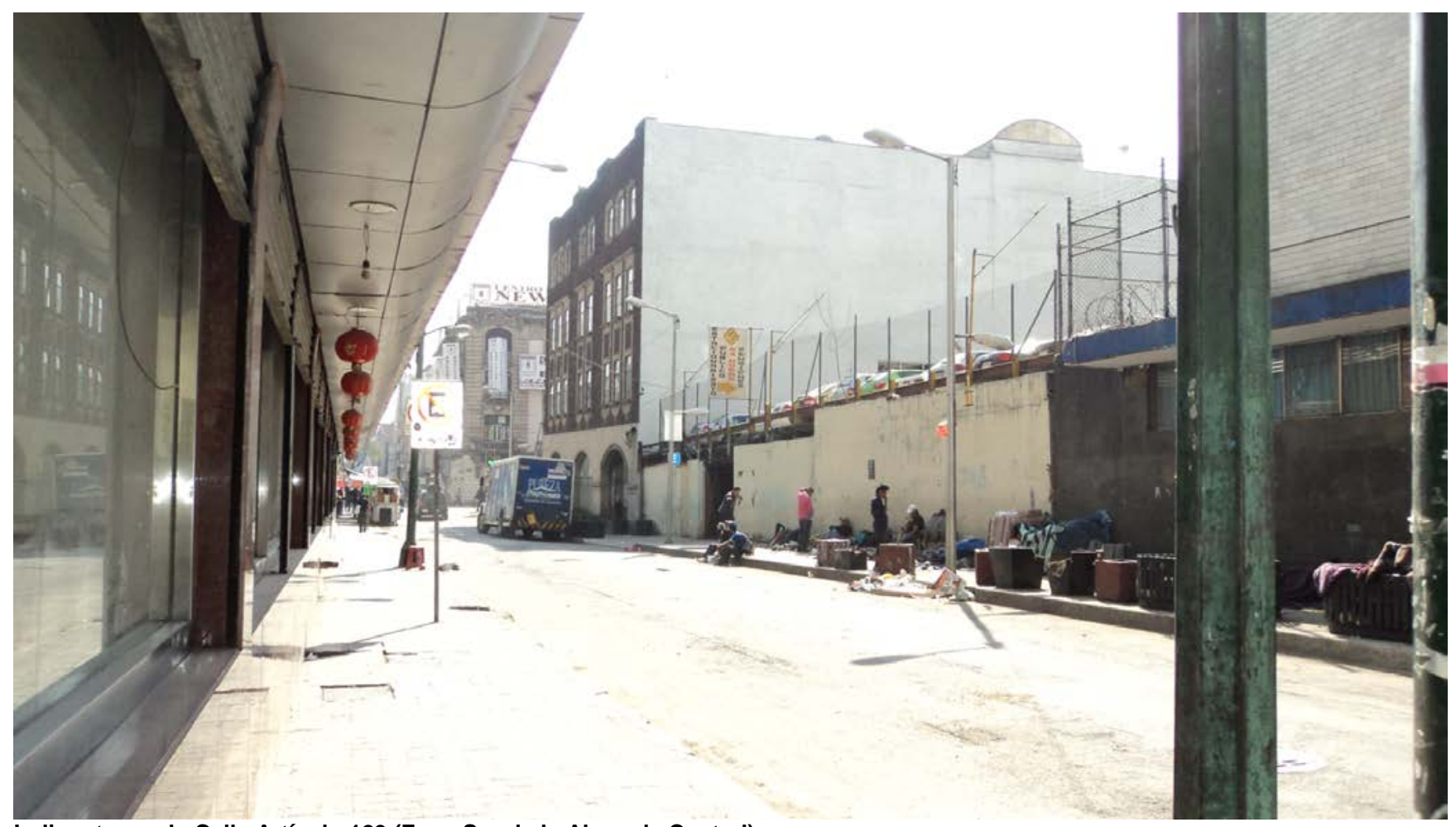

Indigentes en la Calle Artículo 123 (Zona Sur de la Alameda Central)

Fuente: Fotografía del autor, marzo 2016.

La presencia de los indigentes en la zona se podría explicar -al menos- por tres razones: 1. La cercanía a sus sitios de sustento y trabajo: los llamados "indigentes de Artículo 123" son subempleados por los repartidores de los periódicos Excélsior y El Universal -establecidos en esta zona desde 1921 aproximadamente-, para cargar y acomodar las unidades que salen de madrugada de las editoriales a cambio de alimento o droga -no un salario-. Estas personas también se dedican a la recolección de papeles, cartones y periódicos viejos, para venderlos a empresas recicladoras de papel. 2. Por el conflicto existente entre las dependencias gubernamentales y las instituciones de beneficencia pública existentes: la Comisión Nacional de Derechos Humanos -CNDH- que ofrece protección a esta población e impide su desalojo por medio de la fuerza pública, ya que no representa un peligro para la sociedad; y 3 . Aunque en el contexto mexicano es usual que las autoridades negocien -y simulen- los desalojos con los líderes para conseguir apoyo político y enriquecerse de forma ilícita, ese proceso no se puede llevar a cabo con los indigentes, puesto que son personas que viven fuera del sistema y no tiene nada que ofrecer a la corrupción.

\subsection{El caso del Corredor Cultural Regina}

En el Corredor Cultural Regina se llevó a cabo la peatonización de la calle y el incremento de oferta de servicios turísticos, por lo que estas acciones y sus consecuencias fueron interpretadas como parte de procesos de gentrificación. En ciertos estudios realizados en la zona se presentan contradicciones internas y confusión en el planteamiento, como se puede ver a continuación:

En el estudio de Graciela Zamora (2014) titulado: Las políticas de revitalización del Centro Histórico de la Ciudad de México y su contribución al proceso de gentrificación: El caso del Corredor Cultural Regina, la contradicción principal se encuentra en que primero sostiene que hay desplazamiento de población, pero luego sostiene que no existe. El problema de fondo no es la contradicción sino que, a partir de la revisión de múltiples definiciones del concepto, lo reelabora para afirmar la presencia del proceso en el sitio de estudio.

Su planteamiento lo realiza con base en las siguientes definiciones "la gentrificación es un fenómeno que consiste en el cambio socio-espacial por el cual se genera una rehabilitación de la propiedad residencial y un desplazamiento poblacional por parte de una clase social con mayor capacidad económica a la que reside habitualmente en ese espacio. (Pacione 2001:663)" "[...] la gentrificación se considera como un proceso de transformación del espacio urbano que genera cambios en el espacio físico, pero en su complejidad la transformación no se ve reflejada únicamente en el espacio, sino en todas las esferas que 
componen la complicada vida urbana, tales como las relaciones sociales que en él acontecen (Sargatal 2000)". (Zamora 2014:10).

La autora describe, en el sitio de estudio, fenómenos sociales y urbano territoriales ceñidos a la temática de la gentrificación: la brecha de renta, el mejoramiento físico del entorno -la peatonización de la calle-, la atracción de artistas al sitio y su posterior expulsión fomentada por el Gobierno de la ciudad y el sector privado - menciona al inversionista Carlos Slim como uno de los actores principales-, la promoción de actividades culturales y el cambio de perfil socioeconómico de la población flotante, el cambio en las relaciones sociales que se dan en la calle, entre otros; también menciona que:

"El cambio en la composición social del vecindario se ha notado en la llegada de nuevos residentes, sin que ello haya significado la desaparición de las antiguas vecindades o el desplazamiento masivo de antiguos habitantes" (Ibíd. 106); a pesar de lo anterior, la autora afirma la existencia del proceso de gentrificación en el sitio, debido a: "la transformación del carácter de la calle; el tipo de residentes que la habitan que en la actualidad a cambiado a personas de mayor nivel socioeconómico al que solía habitar la zona, y a el [sic.] tipo de establecimientos que solíamos encontrar, hoy en día han sido reemplazados por negocios más rentables"(Ibíd:154).

Cuando la autora indica que la población antigua aún reside en el sitio se implica: 1. Que el desplazamiento y la sustitución de población ya no son los elementos principales que fundamentan su trabajo; o bien, 2. Que la gentrificación no está presente. A pesar de ello, concluye que sí existe el proceso, porque: "[...] la gentrificación hoy en día va a estar enmarcada en un contexto global que hará que las diferentes ciudades respondan a características comunes, pero teniendo particularidades locales condicionadas por los elementos políticos, económicos y culturales propios de cada lugar. Pese a que las fuerzas motoras de la gentrificación diferirán entre ciudades los estudios existentes a lo largo de múltiples ciudades muestran la necesidad de condiciones mínimas para que el proceso ocurra: propiedades o áreas susceptibles de ser gentrificables y a la presencia de actores gentrificadores" (Ibíd:153). ${ }^{5}$

En resumen, esta autora se ve en la necesidad de redefinir el término para obtener los resultados esperados: ello nos muestra que la falta de rigor académico, aunada a la indeterminación del concepto, se puede conducir a la contradicción interna en los estudios. Algo similar sucede con el estudio de la investigadora Ana Valeria Pérez, titulado: "Recuperación de espacios públicos como proceso de gentrificación: aportación desde la perspectiva cultural. Estudio de caso: Corredores peatonales Regina y Madero en el Centro Histórico de la Ciudad de México, 2016"; en él también se indica la presencia de gentrificación en esta zona, aun cuando menciona que no existe desplazamiento de la población original.

Es preciso señalar que la observación directa en el Corredor Cultural Regina no alcanza para sostener la presencia o ausencia de gentrificación; las mejoras en el entorno son evidentes, así como el cambio de giro en los establecimientos comerciales; al mismo tiempo se nota que la población que acude a estos sitios son de perfil socio económico medio y medio bajo, y se ha obtenido información primaria que permite sostener que no hay sustitución de la población original por otra con mayor poder adquisitivo (ver gráfico 3).

\footnotetext{
${ }^{5}$ Lo último nos lleva a preguntar: en el sentido que plantea la autora, ¿no son gentrificables todas las áreas -urbanas o rurales-?, o ¿en cualquier zona no está siempre presente un actor con suficiente poder económico o político que lo haga un gentrificador potencial? Entonces, ¿la gentrificación no está en todas partes?
} 


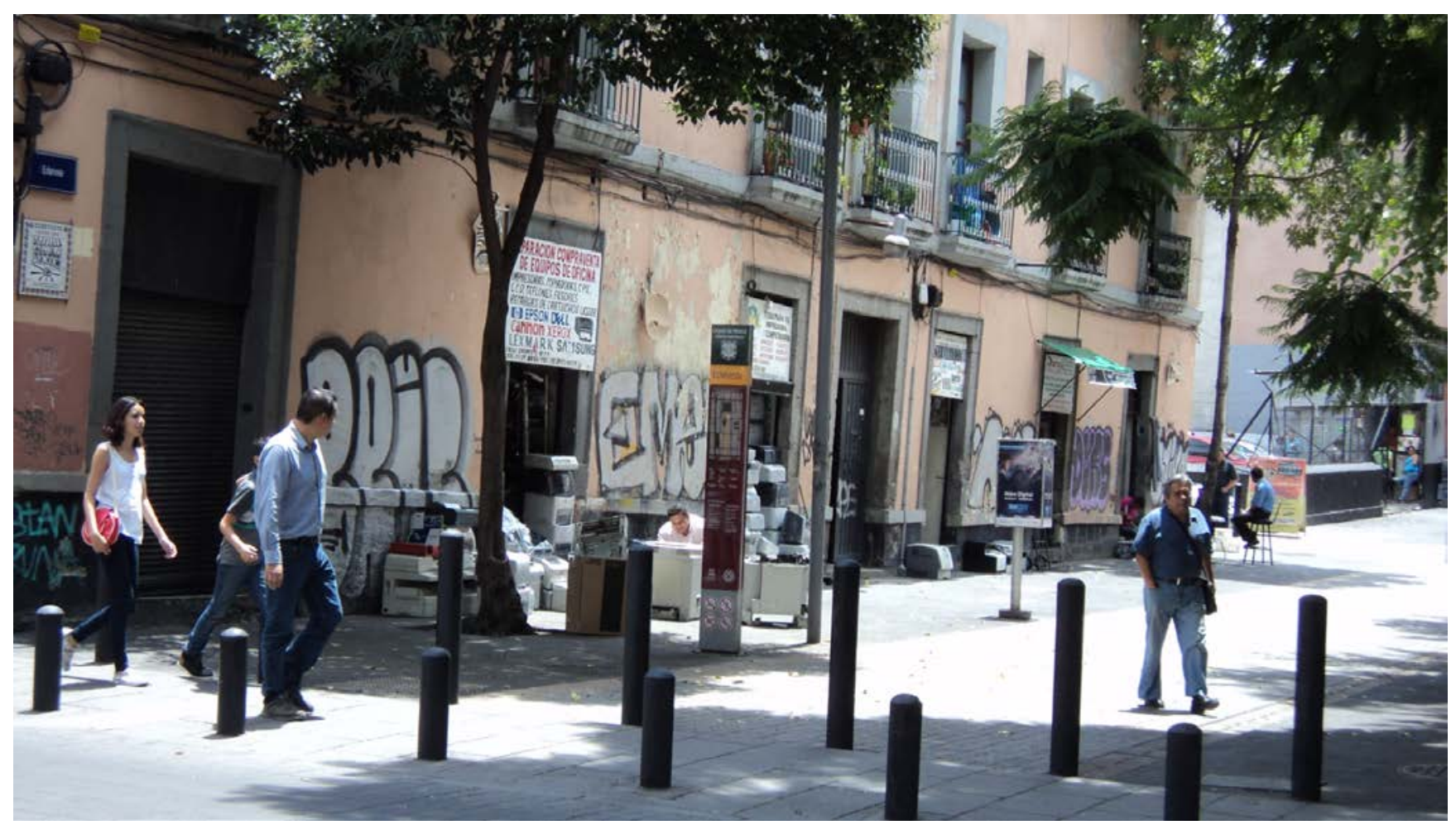

¿Gentrificación en la calle Regina?

Fuente: Fotografía del autor, febrero 2016.

Por lo anterior se concluye que no es pertinente buscar las variables -definidas con anterioridad por otros autores-, para determinar si existe o no gentrificación sin indagar en la realidad concreta del sitio de estudio porque ello no implica que el proceso ocurra y, al generalizar su empleo, se pueden perder de vista otros fenómenos sociales y urbano territoriales que podrían estar presentes.

\section{LAS PROBABLES CAUSAS DEL EMPLEO DEL CONCEPTO GENTRIFICACIÓN EN CIUDAD DE MÉXICO.}

Una vez que el empleo del concepto gentrificación ha sido discutido y se ha mostrado, a nivel local, que los trabajos que abordan la temática presentan irregularidades conviene preguntarse: ¿por qué se incrementa el número de publicaciones sobre el tema en el contexto de Ciudad de México?

La existencia de irregularidades en los estudios ha generado un debate a nivel internacional sobre la pertinencia del empleo del concepto. Hay quienes están a favor de su uso porque ofrece una articulación con otros planteamientos teóricos, y podría ser el detonante de luchas sociales en contra de la urbanización neoliberal (López E., 2016); hay quienes plantean que podría emplearse siempre que exista información sobre el estado anterior al inicio de la gentrificación, para discernir cuáles han sido las modificaciones (Duque, 2010); y finalmente quienes rechazan la adopción del concepto, puesto que no considera las particularidades del contexto, que finalmente definen los procesos sociales (Pradilla, 2013).

Para Maloutas, al trasladar el término fuera de los límites espacio temporales donde fue concebido -Inglaterra, 1964-, tuvo que re enfocarse en tres aspectos: su impacto social, la inversión necesaria para la reorganización de la zona urbana donde se observa, y el papel de los grupos de clase media para mantener el proceso en movimiento (Maloutas, 2011:36). Al trasladarse, habría perdido parte de su especificidad -ser de un lugar y tiempo concretos, responder a ciertas causas y tener determinados efectos- como parte de los procesos de urbanización de las ciudades inglesas donde, por muchos años, se concentraron las industrias, la mano de obra especializada, la vivienda en tugurios, el desarrollo tecnológico y una enorme riqueza.

Sin embargo, y al margen del debate sobre el uso del término, muchos investigadores locales han recibido la influencia de colegas de otros países, han trasladado sus preocupaciones sobre la gentrificación a sus estudiantes y estos a su vez han fortalecido el uso del empleo en el contexto local a través de artículos y tesis. El empleo de tecnologías de la información, y la existencia de redes internacionales de investigadores y colaboradores vinculados a la temática, también son parte de ese proceso de difusión. 
La discusión se ha proyectado fuera del ámbito académico, en un proceso de educación-adoctrinación de la población: colectivos como Left Hand Rotation, vinculado con la red internacional de estudios sobre geografía urbana Contested Cities transmiten el "conocimiento y estrategias internacionales de lucha anti-gentrificación" a la sociedad mexicana y a la academia, mediante congresos, talleres y mesas de trabajo. ${ }^{6}$

Con base en lo último, se presentan tres explicaciones para la difusión del concepto gentrificación en Ciudad de México; 1. Que formamos parte de una sociedad de masas en la que no hay un juicio crítico y es evidente la mercantilización y consumo de conocimiento; 2. Que el concepto forma parte de la reproducción de la ideología hegemónica; 3. Se busca la acreditación en el campo académico para -consciente o inconscientemente- ejercer una posición de poder.

Con respecto a lo primero, para Gustave Le Bon (1895), las masas son una muchedumbre de conducta homogénea, caracterizadas por la irracionalidad, el esquematismo simplista, el dogmatismo, la intolerancia y la credulidad. Otras características que se observan en la sociedad de masas son: la formalización; -en la cual se uniforman y estandarizan las formas expresivas y la difusión masiva de relatos e imágenes a través de las cuales los individuos y colectividades reflejan su identidad; eso explica por qué encontramos estereotipos, esquematismos y simplificaciones, que se convierten en un requisito de la difusión mediática: $\underline{\mathrm{si}}$ un mensaje no es simple, accesible o esquemático, no se propaga;

También están: la pasividad, en la cual los sujetos sociales observamos los acontecimientos actuales como espectadores, mitigando la responsabilidad y la conciencia de participación y colectividad, lo que plantea una crisis de la concepción comunitaria del orden social y una suspicacia respecto al control y manipulación; el aislamiento entre individuos, generado por los desarrollos tecnológicos que facilitan la existencia de espacios públicos en espacios privados; la pérdida de autenticidad: el simulacro y la lógica del "como si", en la que nada parece ser lo que es; la fusión de la realidad con la ficción, que deriva en la producción hacia la espectacularidad y desplazan la atención del significado a la técnica de producción; y la hipervisibilidad en la que la imagen se vuelve un signo intrínseco de la presencia y se vuelca a un desarrollo en la dinámica del deseo: ver equivale a hacer.

En cuanto a la producción de conocimiento este adquiere un rasgo sustancial de utilidad, que conduce a la especialización en alguna temática y/o disciplina. Los criterios de verdad como aplicabilidad, de eficacia como utilidad y de conocimiento como un recurso acumulable generan a su vez la mercantilización y consumo de dicho conocimiento. Para Anthony Giddens (1990) la pérdida de la experiencia es una de las características de la sociedad moderna: es probable que las nuevas generaciones de investigadores basen sus estudios en otros previos sin acercarse a la realidad, o sin la exposición a las vivencias individuales.

Además de la facilidad con que se propaga cualquier concepto novedoso en la sociedad de masas, es posible comprender el proceso de difusión del concepto a partir del planteamiento de Gramsci (1967) con respecto a la reproducción de la ideología hegemónica, entendida como el conjunto de normas culturales y valores impuestos por la clase dominante para prevalecer en el poder.

Este autor explica que, para reproducir su ideología, la clase dominante se vale de tres medios: la educación, la religión y los medios de comunicación, y necesita de la formación de ciertos intelectuales para que cumplan tareas alternas en la hegemonía social. Gramsci los clasifica en tres tipos: 1. los orgánicos, quienes buscan el poder, por lo que obedecen a las clases hegemónicas y se alinean con un partido a través de las publicaciones que realizan, para posteriormente formar parte del poder público y convertirse en los asesores de los líderes; 2. Ios intelectuales libres, quienes no pertenecen a un partido, publican libremente lo que piensan ante la sociedad; y 3 . los intelectuales burócratas, quienes se encargan de elaborar una doctrina y la implementan sin analizar las posibles consecuencias de sus acciones.

\footnotetext{
${ }^{6}$ En septiembre del 2016 este colectivo llevó a cabo el taller: "Gentrificación no es un nombre de Señora" en el cual se explicó a los asistentes -vecinos de las colonias Juárez, Centro y comerciantes del mercado de la Merced, entre otros- algunas de las características generales del concepto gentrificación.

Los vecinos expusieron sus problemáticas concretas: el encarecimiento del alquiler en los inmuebles, la solicitud de desalojo y no renovaciones de contrato por parte de los renteros, el acoso inmobiliario, y la sospecha de que el redesarrollo se vincula al lavado de dinero; los comerciantes temían que las remodelaciones del mercado, propuestas por el Gobierno y el sector privado, culminaran en su expulsión; otros expusieron la forma en que han resistido al desalojo tras 30 años de acoso por parte del Gobierno.

Cuando el colectivo "explicó" el significado de gentrificación, la población afectada lo asumió sin cuestionamientos; inclusive en otros foros vecinales, los comerciantes usaron este concepto para referirse a su problemática.
} 
La legitimidad de los intelectuales orgánicos y burócratas se construye a partir del consenso espontáneo de las grandes masas de la población, pero con la directriz marcada por el grupo dominante; el pensamiento que éstos producen asegura activa o pasivamente la disciplina de los grupos que podrían eventualmente mostrarse en desacuerdo, y son una reserva de legitimidad del poder en previsión de momentos de crisis de mando y dirección. Todos los intelectuales son aparentemente independientes de las clases sociales, pero en realidad mantienen con ellas una estrecha relación.

Por lo anterior conviene cuestionarse: si la difusión del concepto se cumple por medio de los canales institucionales, se sirve de la legitimidad de los académicos para posicionarse entre la población e impone una lectura unívoca de los procesos sociales, ¿no es posible que sirva para la reproducción de la ideología hegemónica y permita a la clase dominante prevalecer en el poder?

Finalmente, es posible que el uso del término tenga relación con la apropiación personal de capital cultural para que sus enunciadores adquieran predominancia social y se inserten en distintos campos desde una posición de poder. Para Pierre Bourdieu las desigualdades sociales se relacionan con la transmisión y apropiación del capital cultural -es decir todos los hábitos, educación, escalas de valor aprendidas-; es probable que los académicos mantengan su posición de poder dentro del campo a través del uso de estos conceptos novedosos, muy difundidos aunque poco estudiados, y obtengan así el reconocimiento y prestigio en su ámbito laboral: las acreditaciones, grados obtenidos, número de publicaciones, asistencia a encuentros académicos, etc., son formas de medir la aceptación social de un individuo dentro de un grupo social.

En este sentido, ¿es posible que el empleo del concepto en el contexto mexicano se deba a la presión que ejercen las instituciones académicas sobre los investigadores para generar artículos científicos que correspondan con lo que se discute en el ámbito internacional y así mantenerse acreditados?, ¿o una respuesta de los investigadores para ascender a una posición respetable en el ámbito académico que les permita sobresalir, adquirir notoriedad y eventualmente, ascender en la escala social?

En México, el Consejo Nacional de Ciencia y Tecnología promueve la producción de artículos científicos mediante estímulos económicos en el Sistema Nacional de Investigadores SNI: con el "objeto de promover y fortalecer, a través de la evaluación, la calidad de la investigación científica y tecnológica, y la innovación que se produce en el país. El Sistema contribuye a la formación y consolidación de investigadores con conocimientos científicos y tecnológicos del más alto nivel como un elemento fundamental para incrementar la cultura, productividad, competitividad y el bienestar social" (CONACYT, 2014). Esta forma de recompensar económicamente la producción científica muchas veces obliga a los investigadores a tratar los temas coyunturales, sin dominar plenamente sus bases teórico-metodológicas, con el fin de obtener estímulos inmediatos, tanto en lo económico como en el prestigio social

Franzoia señala que: "Pierre Bourdieu llamaba doxósofos a aquellos que filosofan a partir de la apariencia de las cosas, es decir que no logran captar las leyes de su funcionamiento interno. [..] En la era del capitalismo globalizado, proliferan los intelectuales que se instalan en el escenario de las apariencias, de las pseudoconcreciones, con lo que cristalizan como auténticos doxósofos. A partir de las apariencias enfocadas desde una filosofía abstracta, construyen conjeturas, que por la fuerza de los intereses de clase que expresan, adquieren el status de teorías "respetables" y a veces "científicas". Desmitificar estas producciones, despojarlas de su halo de conocimiento incuestionable que se correspondería con la supuesta "naturaleza de las cosas", es un ejercicio muy recomendable para comenzar a producir un conocimiento alternativo, propio de naciones, clases y grupos sociales sojuzgados por los globalizadores de la aldea" (Franzoia, 2004).

Miguel Ferreira, basado en las propuestas de Bourdieu sobre los doxósofos hace una observación sobre: "la abundancia de la producción sociológica que, lejos de las preocupaciones de conocimiento que le deberían ser propias, no hace sino reproducir unos esquemas formales, demandados por la "industria de la publicación" científica actual, que transforman lo que debería ser "conocimiento" en mera recopilación de ítems cuantificables según los esquemas bibliométricos al uso. En general, resulta más bien prescindible tomarse la molestia de leer, accediendo a los contenidos de dichos productos de la industria sociológica: lo único relevante es comprobar en qué medida se ajustan o no a los requerimientos formales demandados por la cuantificación bibliométrica" (Ferreira, 2017:143).

\section{CONCLUSIONES}

En este artículo se busca cuestionar la adopción acrítica del concepto gentrificación en el Centro Histórico de Ciudad de México porque, en primer lugar, no alcanza para describir ni explicar los hechos concretos que 
suceden en este contexto, lo que puede incidir en la elaboración de diagnósticos erróneos y la probable adopción de soluciones que no mitiguen la problemática objetiva; en segundo lugar, porque al emplear un concepto ambiguo e indeterminado se genera confusión entre los investigadores, lo que no aporta a la comprensión y solución de los fenómenos urbano territoriales que suceden en la realidad.

Para discutir la pertinencia del empleo del concepto gentrificación en el contexto local en primer lugar se presentó el análisis de publicaciones que abordan la temática, y se detectó que presentan una serie de falencias derivadas de la ambigüedad e indeterminación del término: contradicciones internas en los trabajos, conclusiones con evidencias insuficientes o sin presentar datos contundentes y una serie de limitantes metodológicas para determinar la presencia del proceso.

Se encontró que las publicaciones aluden a fenómenos sociales y urbanos diferentes, ya que desde que se acuñó el concepto se le ha dotado de diferentes significados dependiendo directamente de la postura de los autores, de las variaciones que se han registrado en diversas regiones geográficas, y de la época en que fue estudiado.

En segundo lugar, se presentaron dos casos concretos de estudio donde se observan discrepancias entre los resultados obtenidos por investigadores y los datos recogidos para realizar este artículo. En el primer caso sur de la Alameda Central- se evidencia un proceso opuesto a la denominada gentrificación, ya que las condiciones económicas y sociales del entorno generaron el fracaso de los desarrolladores inmobiliarios; en el segundo caso -Corredor Cultural Regina-, se encontró que, a pesar de la mejora en el entorno, los cambios de uso de suelo y el papel del gobierno para favorecer nuevas actividades sociales, no hay evidencia de sustitución de población.

Finalmente, para explicar el uso masivo del concepto por parte de los investigadores locales se sugieren tres razones: que vivimos una época caracterizada por la irracionalidad, el esquematismo simplista, el dogmatismo, la intolerancia y la credulidad, que permite la difusión y consumo masivo de conceptos novedosos sin que exista reflexión al respecto; que muchos académicos son intelectuales orgánicos y burócratas, instrumentos de la clase dominante para la reproducción de sus normas culturales y valores hegemónicos; que las instituciones presionan a los académicos a entrar en una dinámica competitiva con respecto a la productividad científica, por la cual ganar notoriedad y mejor posición social, aunque sus publicaciones no alcancen a explicar la realidad ni sean la vía para transformarla.

Es por lo anterior que se sugiere que, tanto en la academia como en la sociedad, se fomente la creación de una comunidad con pensamientos autónomos y libres de prejuicios; también es importante retomar la conciencia del papel que cumple un académico dentro de la sociedad.

\section{BIBLIOGRAFÍA}

ATKINSON, R., y BRIDGE, G. (2010). Globalisation and the new urban colonialism. En BROWN - SARACINO, J. The Gentrification Debates (pp. 56-57). NY, EUA: Routledge.

BEAUREGARD, R. (1990, Julio 1). Trajectories of neighborhood change: the case of gentrification. Environment and Planning A: Economy and Space (EUA), 22(7), 855-874.

BOURNAZOU, E. (2015, Enero 02). Cambios socioterritoriales e indicios de gentrificación. Un método para su medición. Academia XXII (México), 12(12), 47-59.

BROWN - SARACINO, J. (2010). The Gentrification Debates. (U. o. Illinois, Ed.) NY, EUA: Routledge.

CORDERO, L. d. (2015). Gentrificación: un análisis visual en el centro histórico de la Ciudad de México . México: Instituto de Geografía UNAM

DAVIDSON, M., y LEES, L. (2005). New-build "gentrification" and London's riverside renaissance. (K. C. Department of Geography, Ed.) Environment and Planning A: Economy and Space (EUA), 37, 11651190.

DÍAZ, I. (2014). La transformación del Centro Histórico de la Ciudad de México. ¿Gentrificación o repoblación? XII Coloquio y Trabajos de Campo del Grupo de Geografía Urbana (AGE) Territorios inconclusos y sociedades rotas. Comunicaciones (p. 10). Madrid: UNAM, Instituto de Geografía. 
DUQUE, R. (2010, Junio 5). La difusión del concepto gentrification en España: reflexión teórica y debate terminológico. Revista bibliográfica de Geografía y Ciencias Sociales (España), 15(875).

FERREIRA, M. (2017). Sobre las incontinencias "doxósofas" del pseudo-conocimiento sociológico. Intersticios: Revista Sociológica de Pensamiento Crítico (España), 11(1), 143-154.

GLASS, R. (1964). London: Aspects of change. En BROWN - SARACINO, J. The Gentrification Debates (pp. 19-30). NY, EUA: Routledge.

GIDDENS, A. (1990). Consecuencias de la modernidad. Version española(1993). (A. Lizón Ramón, Ed.) Madrid, España: Alianza Universidad.

GRAMSCI, A. (1967). La formación de los intelectuales. (A. González Vega, Trans.), México: Grijalbo SA.

GUTIERREZ, G. (2016). Gentrificación en la centralidad de la Ciudad de México: San Miguel Nonoalco y Santiago Tlatelolco. México: Urbanismo UNAM.

HIERNAUX, D., y GONZÁLEZ, C. I. (2014). Gentrificación, simbólica y poder en los Centros Históricos: Querétaro, México. XIII Coloquio Internacional de geocrítica, El control del espacio y los espacios de control, España, 5-12 mayo: Universidad de Barcelona.

LEES, L. (2003). Super-gentrification: The Case of Brooklyn Heights, New York City. En BROWN SARACINO, J. The Gentrification Debates (pp. 45-50). NY, EUA: Routledge.

LEES, L., SLATER, T., y WYLY, E. (2008). Gentrification. NY, EUA: Routledge.

LÓPEZ, E. (2016, 11). Acerca de una gentrificación "planetaria", políticamente útil. INVI (Chile),31(88), 217240.

LÓPEZ, N. S. (2016). Soy de la guerrero y aquí ¿me quedo?: entre el derecho a la ciudad y la gentrificación. México: Facultad de Arquitectura UNAM.

MALOUTAS, T. (2011). Contextual Diversity in Gentrification Research. Critical Sociology (Reino Unido), I(38), 33-48.

MARTíNEZ, U. (2014). Mercantilización de los espacios públicos dentro del proceso del gentrificación del centro histórico de la Ciudad de México:el caso de la Alameda Central . México: Instituto de Geografía UNAM.

MONDRAGÓN, M. (2015). Proceso de gentrificación de la colonia Roma . México: Facultad de Arquitectura UNAM.

OLIVERA, P., y DELGADILLO, V. (2014, septiembre). Políticas empresarialistas en los procesos de gentrificación en la Ciudad de México. Revista de Geografía Norte Grande (Chile),(58), 111-133.

OLVERA, M. (2016, febrero 24). Relato de entrevista sobre proyecto Living Centro Histórico. (I. Garza Rodríguez, entrevistador) México.

PÉREZ, A. V. (2016). Recuperación de espacios públicos como proceso de gentrificación: aportación desde la perspectiva cultural. Estudio de caso: Corredores peatonales Regina y Madero en el Centro Histórico de la Ciudad de México. (D. J. Martínez Reséndiz, Ed.) México: Facultad de Filosofía y Letras UNAM.

PRADILLA, E. (2013, Mayo- Agosto). América Latina Urbana: La construcción de un pensamiento teórico propio. Entrevista con Emilio Pradilla Cobos. (V. Delgadillo, Entrevistador): Andamios (México)10(22), 47-59.

RODRÍGUEZ, I. J. (2016). Gentrificación en San Sebastián Xoco, Delegación Benito Juárez, Distrito Federal . México: Insttituto de Geografía UNAM. 
RODRÍGUEZ, V., y PUGA, D. y. (2002). Bases para un estudio de la gentrificación en Madrid. En Boletín de la Real Sociedad Geográfica (Vols. Tomo CXXXVII-CXXXVIII , pp. 273-310). España: Real Sociedad Geográfica.

ROMERO, E. (2017). Gentrificación en la Ciudad de México: el caso de la Colonia Juárez. México: Instituto de Geografía UNAM.

SALINAS, L. A. (2013). La gentrificación de la Colonia Condesa, Ciudad de México. Aporte para una discusión desde Latinoamérica. Revista Geográfica de América Central, 2(51), 145 -167.

SMITH, D., y PHILLIPS, D. (2001). Socio-cultural representations of greentrified Pennine rurality. (U. o. School of Geography, Ed.) Journal of Rural Studies (EUA), 17, 457-469.

SMITH, N. (1979). Toward a Theory of Gentrification: A Back to the City Movement by Capital not People. (A. Journal, Ed.) Journal of the American Planning Association (EUA), 4(45), 538-548.

SMITH, N. (1998). A short history of gentrification. En BROWN - SARACINO, J. The Gentrification Debates (pp. 31-37). NY, EUA: Routledge.

TORRES, M. E. (2017). Diseño y gentrificación: el papel del diseño gráfico en la escenografía del centro urbano. México: Diseño y Comunicación visual UNAM.

UNAM. (2017). Dirección General de Bibliotecas. Consulta Gentrificacion. (U. N. México, Ed.) México.

VÁZQUEZ, C. (1996). La gentrificación. Un modelo de segregación socioespacial en ciudades postindustriales. Universidad Autónoma de Madrid, Departamento de Geografía Humana. España: UAM.

ZAMORA, G. (2014). Las políticas de revitalización del Centro Histórico de la Ciudad de México y su contribución al proceso de gentrificación: El caso del Corredor Cultural Regina. (F. d. Sociales, Ed.) México: UNAM.

Fuentes electrónicas:

CONACYT. (2014). Sistema Nacional de Investigadores, www.conacyt.gob.mx http://www.conacyt.gob.mx/index.php/el-conacyt/sistema-nacional-de-investigadores. Consultado el: 31/03/17

FRANZOIA, A. (2004). Equipo de Investigaciones "Rodolfo Walsh", www.rodolfowalsh.org: www.rodolfowalsh.org: http://www.rodolfowalsh.org/spip.php?article184 Consultado el: 31/03/17

LE BON, G. (1896, 2001). www.socialsciences.mcmaster.ca. Kitchener, 2001. https://socialsciences.mcmaster.ca/ econ/ugcm/3/l3/lebon/Crowds.pdf Consultado el: 15/06/17

TELLEZ, L. F. (2014, abr-jun). La revitalización del Centro Histórico de la Ciudad de México: entre la voluntad de la élite y la realidad del pueblo. Pacarina del Sur Revista de Pensamiento Crítico Latinoamericano (en línea) 5(19), 1-14. http://pacarinadelsur.com http://pacarinadelsur.com/home/mascaras-e-identidades/949-la-revitalizacion-del-centro-historicode-la-ciudad-de-mexico-entre-la-voluntad-de-la-elite-y-la-realidad-del-pueblo.

Consultado el: 12/04/17 